\title{
Molecular Dynamics Simulation Study of Nonconcatenated Ring Polymers in a Melt: II. Dynamics
}

\author{
Jonathan D. Halverson, ${ }^{1}$ Won Bo Lee, ${ }^{1,2}$ Gary S. Grest, ${ }^{3}$ Alexander Y. Grosberg, ${ }^{4}$ and Kurt Kremer ${ }^{1}$, a) \\ 1) Max Planck Institute for Polymer Research, Ackermannweg 10, 55128 Mainz, \\ Germany \\ ${ }^{2)}$ Department of Chemical and Biomolecular Engineering, Sogang University, Shinsu-dong 1, Mapo-gu, Seoul, \\ Korea \\ 3) Sandia National Laboratories, Albuquerque, NM 87185, USA \\ ${ }^{4)}$ Department of Physics, New York University, 4 Washington Place, New York, NY 10003, \\ $U S A$
}

(Dated: 13 October 2018)

\begin{abstract}
Molecular dynamics simulations were conducted to investigate the dynamic properties of melts of nonconcatenated ring polymers and compared to melts of linear polymers. The longest rings were composed of $N=1600$ monomers per chain which corresponds to roughly 57 entanglement lengths for comparable linear polymers. The ring melts were found to diffuse faster than their linear counterparts, with both architectures approximately obeying a $D \sim N^{-2.4}$ scaling law for large $N$. The mean-square displacement of the center-of-mass of the rings follows a sub-diffusive behavior for times and distances beyond the ring extension $\left\langle R_{g}^{2}\right\rangle$, neither compatible with the Rouse nor the reptation model. The rings relax stress much faster than linear polymers and the zero-shear viscosity was found to vary as $\eta_{0} \sim N^{1.4 \pm 0.2}$ which is much weaker than the $N^{3.4}$ behavior of linear chains, not matching any commonly known model for polymer dynamics when compared to the observed mean-square displacements. These findings are discussed in view of the conformational properties of the rings presented in the preceding paper ${ }^{1}$. [DOI: 10.1063/1.3587138]
\end{abstract}

\section{INTRODUCTION}

Understanding the static and dynamic properties of ring polymer melts is one of the remaining challenges in polymer science. Unlike linear polymers, the topological constraints for ring or cyclic polymers are permanent and this affects both their static and dynamic properties. For linear polymers the topological constraints imposed by the non-crossability of the chains (entanglements) force each chain to diffuse along its own coarsegrained backbone, the so-called primitive path, and this is well described by the reptation model of de Gennes and Edwards. For branched systems strands have to fold back in order to find a new conformation without crossing any other chain, resulting in an exponential growth of the longest relaxation time due to the entropy barrier of $\mathcal{O}$ (strand length) between different states $^{2}[4$. A number of simulation and experimental results confirm this concept $\mathrm{t}^{5-13}$. For both linear and branched polymer melts, it is the free chain ends that make the known relaxation mechanisms possible. However, in the case of rings there are no free ends. This raises a number of unanswered questions regarding the motion and stress relaxation of ring polymer melts.

In the companion paper ${ }^{1}$ we discussed the difficulties of understanding the equilibrium (static) properties of a melt of nonconcatenated ring polymers. We pointed out

\footnotetext{
a) The following article has been accepted by The Journal of Chemical Physics. After it is published, it will be found at http: //jcp.aip.org Corresponding author e-mail: kremer@mpipmainz.mpg.de
}

that this system presents a formidable challenge to the current polymer theories. It is of considerable current experimental interest 14 , 20 , and it is believed to have a significant projection on the problem of chromatin structure and dynamics in chromosomes. We refer the reader to the detailed discussion of these issues in the Introduction section of our paper ${ }^{1}$; the large bibliography of relevant references is also in that paper. The difficulty of the melt of rings problem is such that even the equilibrium structure of nonconcatenated rings is highly non-trivial. We have computed a large number of equilibrium characteristics of this system in the previous paper ${ }^{1}$.

In the present paper, the second in a series of two, we investigate the dynamic properties of ring polymer melts using molecular dynamics (MD) simulation. To our knowledge the only previous MD studies of ring polymer melts are by Hur et al. ${ }^{21}$ and Tsolou et al. ${ }^{22}$ who considered polyethylene for short chain lengths (i.e., $N \lesssim 3 N_{e \text {,linear }}$ ), and Hur et al ${ }^{23}$ for chains up to about $11 N_{e \text {,linear, where }} N_{e \text {,linear }}$ is the entanglement chain length of linear polymers at the same density. Here we consider rings and linear melts composed of much longer chains. The number of monomers per ring ranges from

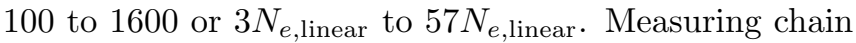
lengths in terms of $N_{e}$,linear provides a basis to compare rather different simulational as well as experimental studies. The linear chain melts were simulated using the same model as the rings with chain lengths varying from 100 to 800 monomers per chain. Such highly entangled melts have very long relaxation times and a highly parallelized simulation code had to be used. For the longest rings as many as 2048 IBM Blue Gene/P cores were used for a single simulation. 
All details about the model and the setup of the systems can be found in the preceding paper, where the very same simulations have been analyzed in order to understand the static properties of nonconcatenated ring polymers. All properties reported here are exclusively taken from the fully equilibrated part of the data. The simulation model is briefly presented in the next section. This is followed by a section on the dynamics as well as a primitive path analysis ${ }^{24}$ of the ring systems. A comprehensive discussion of the results is given in Section IV with the key findings of the work and future challenges presented in the Conclusions section.

\section{SIMULATION METHODOLOGY}

Based on the Kremer-Grest (KG) model10, all beads interact via a shifted Lennard-Jones potential (WCA or Weeks-Chandler-Anderson potential) with a cutoff of $r_{c}=2^{1 / 6} \sigma$. Nearest neighbor beads along the chain in addition interact via the finitely extensible nonlinear elastic (FENE) potential, and chain stiffness is introduced by an angular potential! ${ }^{[3]}$ The model parameters are the same for all ring and linear polymer simulations reported in this work.

The natural time scale of the WCA potential is $\tau=$ $\sigma \sqrt{m / \epsilon}$, where $m$ is the mass of a monomer. For the simulations we use $T=1.0 \epsilon / k_{B}$ and $\rho=0.85 \sigma^{-3}$. This model has been frequently used for simulation studies of polymer melts and solutions, so that one can refer to ample information throughout the discussion of the present, new results. Also, the linear chain data presented in this work is all new. The ring melts were simulated using 200 polymers of length 100 to 1600 monomers per chain. The linear systems were composed of 2500 chains of length $N=100,250$ chains of $N=200$, and 400 chains of length $N=400$ and 800. An important parameter for our discussion is the entanglement length for linear polymers in a melt. For the model employed here this parameter was determined $\sqrt{13}$ to be

$$
N_{e, \text { linear }}=28 \pm 1 \text {. }
$$

For more details of the model, the setup and the notation and software used see the preceding paper ${ }^{1}$.

\section{DYNAMICS RESULTS}

\section{A. Mean-Square Displacements of Monomers and Chains}

The dynamics of ring polymers pose special problems for a theoretical description. Based on the non-trivial structural properties found in the preceding paper we have to anticipate dynamic properties not observed in other polymer systems. While for short rings one can expect that the Rouse model might describe the dynamics and stress relaxation fairly well, this problem for longer rings is completely open. From the static properties one sees that for large $N$ the rings exhibit an $N$-independent intrachain density, $\rho_{\text {self }}$, resulting in a scaling of the overall extension as a globule, while at the same time there is still significant interpenetration between rings. For a dense, unknotted and nonconcatenated globule one could expect a Rouse-like dynamics for the beads on short time scales and an overall diffusion coupled to amoeba-like shape fluctuations, similar to segregated chains in two dimensions 5226 . However, the mutual interpenetration of rings results in a stronger interchain coupling of the motion. Whether there are entanglement effects as in linear polymers is not clear since there are no free ends to reptate. Kapnistos et al ${ }^{[19}$ and Milner and Newhal ${ }^{27}$ have developed models for stress relaxation based on a lattice animal picture. This however requires entropically unfavorable double-stranded conformations, which are only somewhat close to the conformations corresponding to the lattice animal scaling regime of Fig. 2 in the previous paper ${ }^{1}$. Thus since the expected behavior is quite unclear, we present here a rather comprehensive first analysis of single and collective ring polymer dynamic properties. The motion of the rings and linear systems is characterized by the various mean-square displacements (MSD), and for the rings these results do not provide a consistent picture in terms of the known theoretical models.

The first quantities to look at are the different meansquare displacements of monomers and whole chains as a function of time. The MSD averaged over all the monomers of a chain is $g_{1}(t)$, the MSD of the monomers with respect to the center-of-mass of the chain is $g_{2}(t)$, and the MSD of the center-of-mass, $\boldsymbol{r}_{C M}$, of the chains is $g_{3}(t)$ :

$$
\begin{aligned}
& g_{1}(t)=\left\langle\left|\boldsymbol{r}_{i}(t)-\boldsymbol{r}_{i}(0)\right|^{2}\right\rangle, \\
& g_{2}(t)=\left\langle\left|\boldsymbol{r}_{i}(t)-\boldsymbol{r}_{C M}(t)-\boldsymbol{r}_{i}(0)+\boldsymbol{r}_{C M}(0)\right|^{2}\right\rangle, \\
& g_{3}(t)=\left\langle\left|\boldsymbol{r}_{C M}(t)-\boldsymbol{r}_{C M}(0)\right|^{2}\right\rangle .
\end{aligned}
$$

In Eqs. (2) and (3), $\boldsymbol{r}_{\boldsymbol{i}}$ is the position of monomer number $i$ in space.

For linear polymers $g_{1}(t)$ and $g_{2}(t)$ are averaged over the middle five beads to reduce chain end effects 10 . In all cases the diffusion of the center-of-mass of the whole simulation system is subtracted. Fig. 1 shows $g_{1}(t)$ and $g_{3}(t) N$ for the ring and linear systems. Rouse and reptation theory provide a reasonably good explanation for the behavior of the linear systems 4 .10. For the mean-square displacement of the monomers, $g_{1}(t)$, the reptation concept predicts in its asymptotic form ${ }^{4}$ a sequence of power laws of $g_{1}(t) \sim t^{1 / 2}, t^{1 / 4}, t^{1 / 2}, t^{1}$. For short times the standard Rouse behavior dominates. After reaching the Rouse relaxation time $\tau_{e}$ of an entanglement length $N_{e}$ the constraints imposed by the surrounding chains cause the beads to follow a Rouse motion along the random 

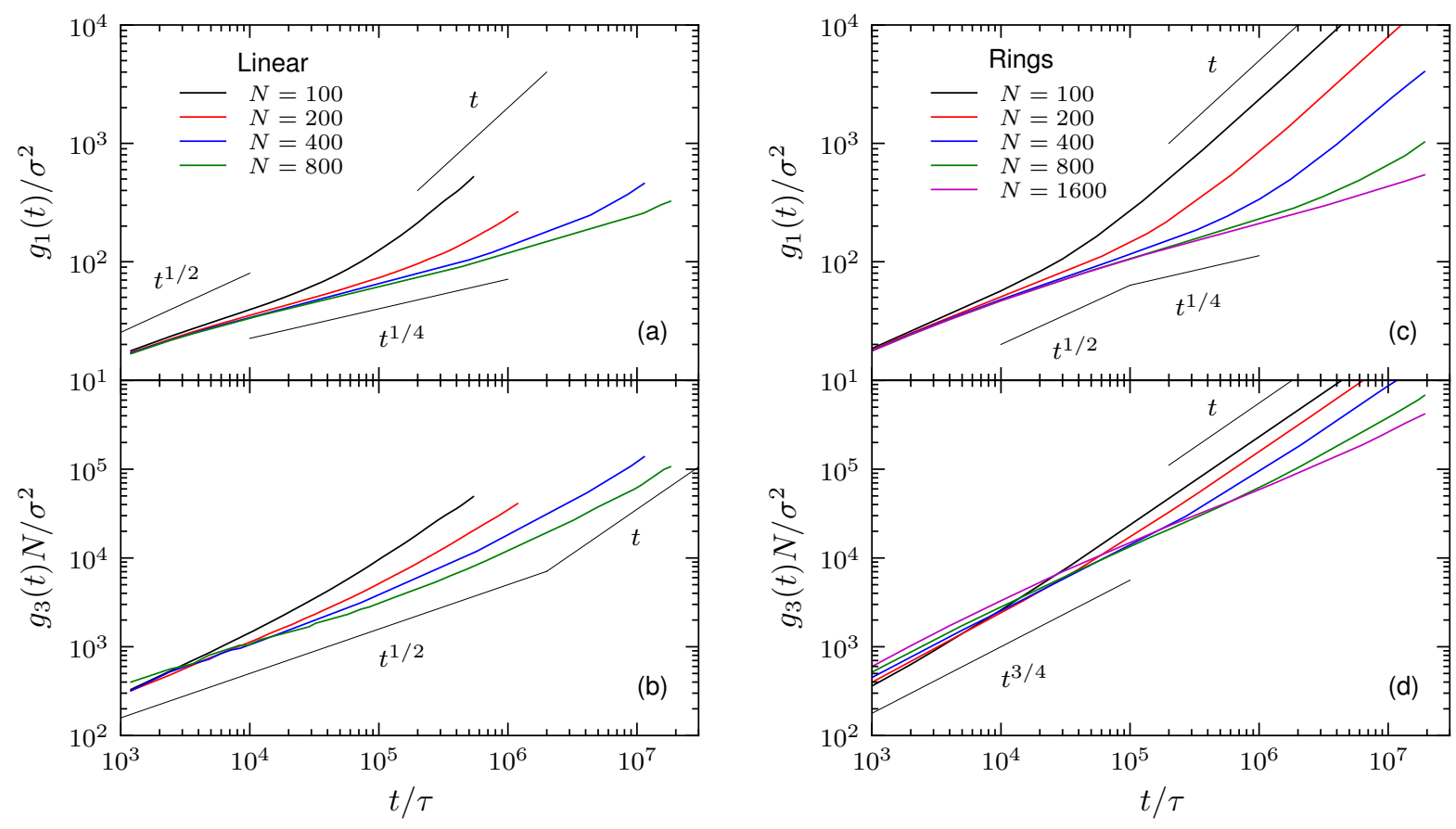

FIG. 1. (a) $g_{1}(t)$ and (b) $g_{3}(t) N$ versus time for the linear systems. These quantities are shown for the rings in (c) and (d). The expected scaling behavior from Rouse and reptation theory is shown along with the $t^{3 / 4}$ regime for the rings.

walk contour of the reptation tube, resulting in a $t^{1 / 4}$ power law. After the Rouse relaxation time $\tau_{R} \sim N^{2}$ the whole chain diffuses along the tube contour, resulting in the second $t^{1 / 2}$ regime followed by the free diffusion in space after the chain leaves the tube, in the ideal model at time $\tau_{d} \sim N^{3} / N_{e, \text { linear. }}$. This intermediate slowing down of $g_{1}(t) \sim t^{1 / 4}$ comes along with a sub-diffusive regime $g_{3}(t) \sim t^{1 / 2}$ for the displacement of the chain centerof-mass. It should however be noted that the expected $g_{3}(t) \sim t$ in the early Rouse regime typically reduces to a $t^{0.8}$ power law due to correlation hole effects.

Fig. 1a,b gives the results for the linear polymer melts for chains up to $N=800$. With an estimated $N_{e, \text { linear }}=28 \pm 1$ we expect a well developed reptationlike behavior for the longer chains. We observe a Rouselike behavior for the shorter chains and a clear intermediate regime in $g_{1}(t)$ with an exponent close to $t^{0.25}$ separating the two $t^{1 / 2}$ regimes in $g_{1}(t)$ for the long chains. There is the well known very smooth crossover, making it very difficult to precisely identify the crossover times for the different regimes. Fig. $1 \mathrm{p}$ shows $g_{3}(t) N$ versus time for the linear systems. As expected from the Rouse description there is an immediate crossover towards free diffusion for the shorter chains whereas the longer ones exhibit a well-defined $t^{1 / 2}$ regime before crossing over into free diffusion (with some small deviations for short times for $N=800$ ). In all cases the free diffusion of the centerof-mass of the chains sets in long before $g_{3}(t)$ reaches the radius of gyration squared, i.e., at about $\left\langle R_{g}^{2}\right\rangle / 2.5$ for $N=200$ and $\left\langle R_{g}^{2}\right\rangle / 3.0$ for $N=800$. Though there are still many details to be understood in the context of the dynamics of linear polymers, the Rouse and reptation theories give a very good semi-quantitative description.

While for linear polymer melts the basic dynamic concepts are fairly clear, the situation for ring melts is strikingly different. Fig. 11p shows $g_{1}(t)$ for the rings. All five data sets follow the same curve during early times. Then, independent of $N$ we observe a significant slowing down in the mean-square displacement from the initial $t^{1 / 2}$ scaling to a power law very close to the $t^{1 / 4}$ expected for perfect reptation of linear polymers. Note however that the rings cannot entangle in the classical sense and thus the rings are not expected to show reptation-like behavior. A scaling regime of $g_{1}(t) \sim t^{0.35}$ has been reported for melts of polyethylene rings ${ }^{23}$ of

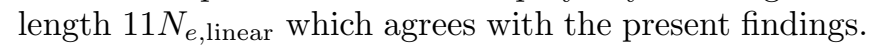
After the approximate $t^{1 / 4}$ regime we do not observe a second $t^{1 / 2}$ regime in $g_{1}(t)$ at a value of $\mathcal{O}\left(N^{1 / 2}\right)$ (much smaller than the radius of gyration squared), but rather a direct crossover toward the diffusion of the whole chain. From Fig. 11k we observe this crossover at a displacement significantly larger than $\left\langle R_{g}^{2}\right\rangle^{1 / 2}$, something not observed in any other polymeric system! The crossover times are around $t \approx 7.6 \times 10^{4} \tau, 2.4 \times 10^{5} \tau, 1.5 \times 10^{6} \tau, 8.2 \times 10^{6} \tau$ and $g_{1}(t) \approx 150 \sigma^{2}, 200 \sigma^{2}, 330 \sigma^{2}, 450 \sigma^{2}$ for $N=100$, 200, 400 and 800, respectively. For the $N=1600$ case, no onset of a crossover towards diffusion is seen for up to $2.0 \times 10^{7} \tau$. In Fig. $1 \mathrm{~d}, g_{3}(t) N$ is shown for the rings along with a reference line with a slope of $3 / 4$. This scaling behavior is seen to provide reasonable agreement with the center-of-mass motion of all five ring systems and for the $N=400$ system it holds for several decades. The 

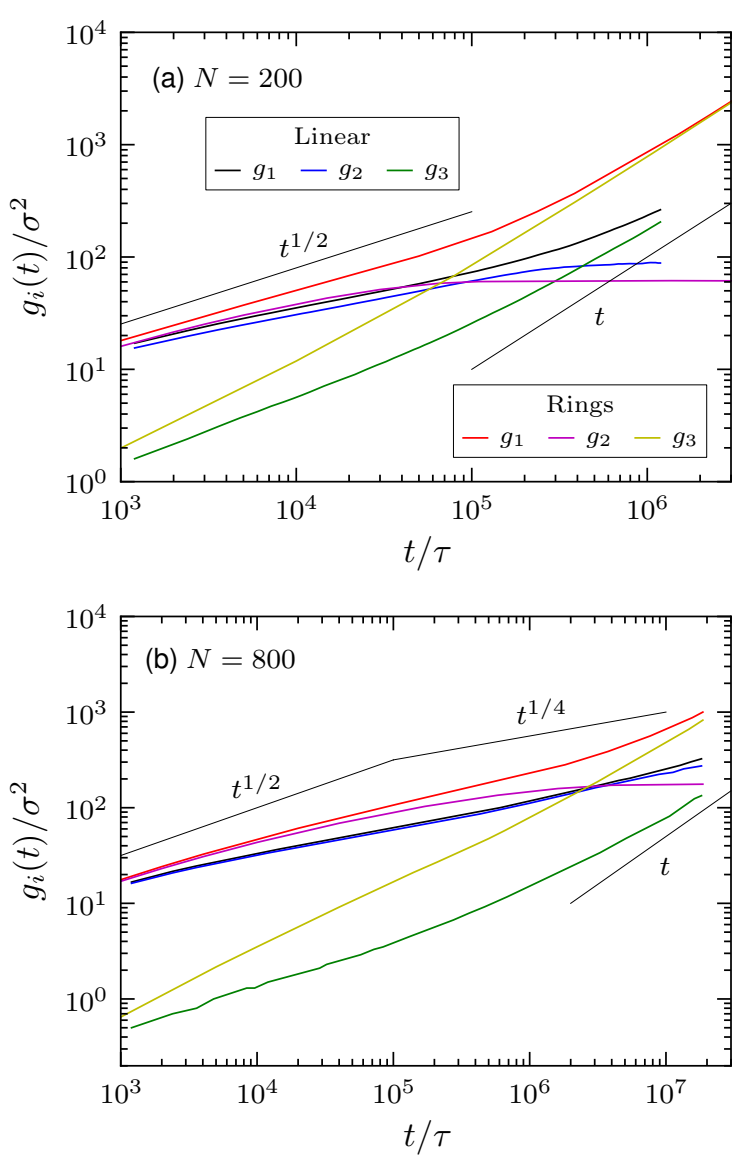

FIG. 2. The mean-square displacements are shown as a function of time for the rings and linear systems with $N=200$ and 800 . The inner monomers are used for the calculation of $g_{1}(t)$ and $g_{2}(t)$ for the linear chains. The scaling laws from Rouse and reptation theory are also shown.

$t^{3 / 4}$ power law reminds one of a correlation hole effect for Rouse chains, rather than any reptation phenomenon. The crossover towards free diffusion is in good agreement to what is observed from $g_{1}(t)$. However, the crossover to the free diffusion time scaling of $t^{1}$ occurs at values significantly larger than the chain extensions (cf. Table 2 of the preceding paper), similar to what is seen for $g_{1}(t)$. This is in contrast to the linear systems which show a $t^{1}$ scaling after moving even somewhat less than their own size. For the longest rings the fully diffusive regime is not reached within the time frame of the simulation. For $N=1600$ eventually we observe an even smaller effective power law, deviating from the $t^{3 / 4}$ towards a minimal power around $t^{0.65}$. The mean-square displacement data for the rings and linear systems is available in the supplementary material ${ }^{28}$.

Another striking observation from Fig. 1 $1 \mathrm{~d}$ is that $g_{3}(t) N$ for short times does not become independent of $N$. It displays a small but still clearly visible ring length dependence, indicating a delayed onset of the constraints imposed by surrounding rings with increasing ring size.
Such phenomena have not been observed in a polymeric system so far and suggests long-range ring-ring correlations, even though the chains cannot be entangled in the classical sense. The slight increase of $g_{3}(t) N$ for short times and increasing $N$ is indicative of the fact that crumpled globules, which is the presumed state of nonconcatenated rings in the melt, are characterized by a rather deep correlation hole $\mathrm{e}^{29}$.

To gain additional insight into these correlations we examined the dynamics of the exchange of neighbors. The neighbors of a given chain are defined as the other chains whose center-of-mass is within a distance $\left\langle R_{e}^{2}\right\rangle^{1 / 2}$ of the center-of-mass of the reference chain. In the preceding pape ${ }^{1}$, we denoted the average equilibrium value of this quantity as $K_{1}(r)$ with $r=\left\langle R_{e}^{2}\right\rangle^{1 / 2}$ or the root-meansquare spanning distance between beads $N / 2$ apart along the ring. At any given time each chain has a specific set of neighbors with the average number of chains in the set being $K_{1}$, which is 11.6 for $N=100$ and 16.7 for $N=1600$. We computed the number of remaining (unexchanged) neighbors after the reference ring moved its own root-mean-square gyration radius. This number was found to increase with increasing chain length from 5.5 for $N=100$ to 7.3 for $N=1600$. We also computed how far the reference chain moved before all neighbors but one were exchanged. The calculation was performed using a large number of time origins and the root-mean-square displacement was found to increase with increasing chain length from about $2.5\left\langle R_{g}^{2}\right\rangle^{1 / 2}$ for $N=100$ to significantly more than $3\left\langle R_{g}^{2}\right\rangle^{1 / 2}$ for $N=800$. It is interesting to note that these displacements are roughly the same as those required for the systems to reach the fully diffusive regime.

Even though the ring polymers display a remarkable and so far unseen slowing down compared to a Rouselike motion, they diffuse significantly faster than their linear counterparts. This is illustrated in Fig. 2, where the MSD for two chain lengths are compared directly for both melts of rings and of linear chains. As expected $g_{2}(t)$ and $g_{3}(t)$ of the rings cross around $2\left\langle R_{g}^{2}\right\rangle$. For the longer rings, however, $g_{3}(t)$ at this crossing point has not yet reached the fully diffusive regime. For the linear polymers the entanglement effects are much stronger, and the crossing of $g_{2}(t)$ and $g_{3}(t)$ is hardly reached. It should however be noted that this crossover for the linear chains is below $2\left\langle R_{g}^{2}\right\rangle$ and occurs in a regime where $g_{3}(t)$ already displays the standard diffusion behavior. The self-diffusion coefficients, as determined by the long-time behavior of $g_{3}(t)$, are shown in Fig. 3 for the rings and linear systems. The rings diffuse faster than the linear systems for all chain lengths considered. This is in agreement with previous simulation result $\$ 30+32$. And while early experimental papers ${ }^{16 / 18}$ report that the two architectures have similar diffusivities, recent experiments cast doubt on these finding $\$ 19$. Reptation theory predicts for linear polymers $D \sim N^{-2}$ for $N>N_{e, \text { linear }}$, however, in practice the observed scaling ${ }^{33}$ is $N^{-2.3 \pm 0.1}$ and this 


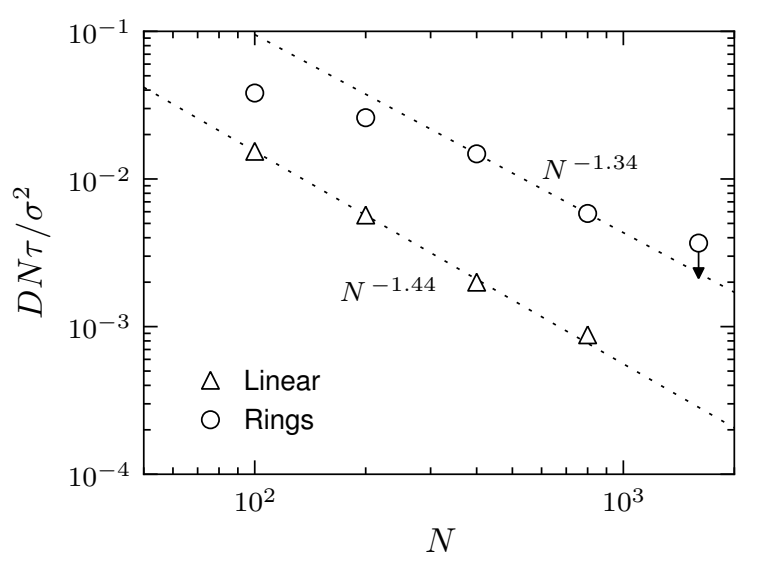

FIG. 3. Self-diffusion coefficient multiplied by $N$ versus chain length for the rings and linear melts. The line through the ring data was fit to the values for the $N=400$ and 800 systems while for the linear data all points were used. The arrow beneath the point for the largest ring system indicates that its value is expected to decrease somewhat until the system reaches the fully diffusive regime. The range of this shift (length of arrow) is based on the extrapolation of $g_{3}(t) / t$ to infinite time. With the exception of the $N=1600$ ring case, all error bars are smaller than the size of the symbols.

is found here to within the error bars. The data for the ring systems are almost parallel (in log-log scale), but shifted to significantly faster diffusion. Using the values from the $N=400$ and 800 cases one finds $D \sim N^{-2.3}$ for the rings. This is a stronger scaling than reported previously for shorter rings. One reason for this is that the present study uses longer chains with $N / N_{e, \text { linear }} \approx 57$ for $N=1600$. Despite a simulation time of more than $3.9 \times 10^{7} \tau$, the longest ring system did not reach the fully diffusive regime. The arrow in Fig. 3 indicates the extrapolated final value which will be slightly lower than the data point. Because the linear systems follow the reptation picture for chains as short as $N=100$, the effective entanglement length for the rings must be higher than that of the linear chains, if the data were to be interpreted in terms of some as yet unknown generalization of the reptation concept. The corresponding entanglement length for the rings, $N_{e, \text { rings }}$, will be estimated in Section IIIB. This is all to be compared to a visualization of the chain motion, which significantly differ between the two architectures. Fig. 4 compares the motion of a linear chain to that of a ring for times less than $\tau_{R}$. The linear chain is found to be largely confined to a tube with the end monomers having more freedom than the inner monomers. This is in sharp contrast to the ring in Fig. 4b which is found to trace out a roughly spherical volume.

The slow crossover towards free diffusion of the rings is quite striking. For melts of strictly two-dimensional polymers it is known that the chains segregate and form 2 -d globular structures. The diffusion is mostly due to

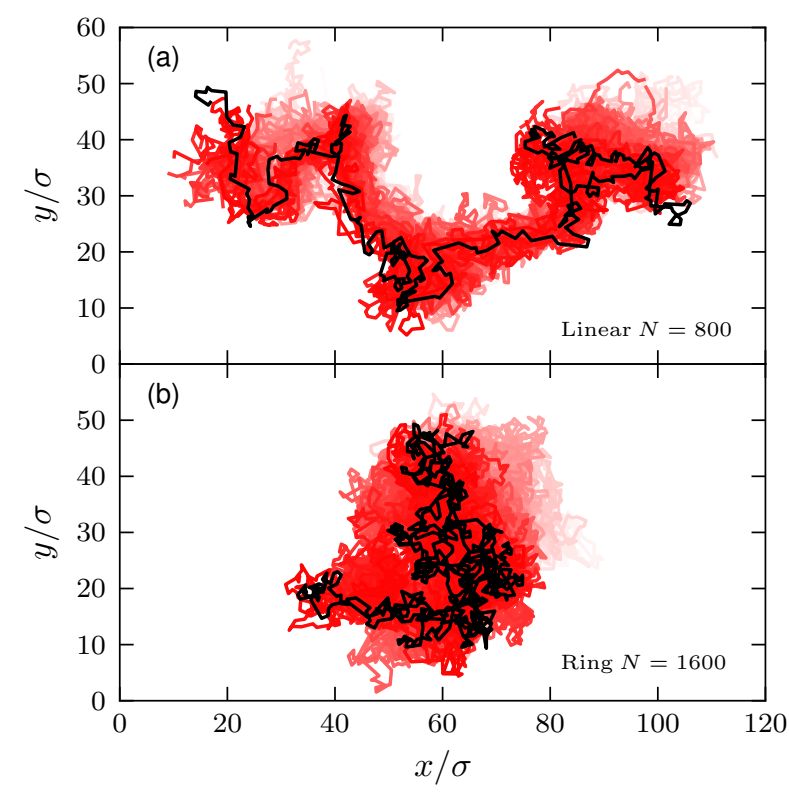

FIG. 4. (a) Linear chain with $N=800$ shown every $12000 \tau$ for 60 conformations or $0.28 \tau_{R \text {,linear }}$ and (b) ring with $N=$ 1600 shown every $10000 \tau$ for 60 conformations or $0.23 \tau_{R \text {,rings }}$. Chain snapshots at later times are drawn darker in color with the final chain conformation in black.

amoeba-like shape fluctuations, which do not require the complete reorganization of the internal winding of the chain. In a similar way we can check whether the diffusion time of a ring, the correlation time of the radius of gyration squared and the internal contour diffusion follow the same time dependency or not. If $\boldsymbol{a}$ and $\boldsymbol{b}$ are spanning vectors whose tails are separated by $N / 4$ monomers (see inset of Fig. 5) then $\boldsymbol{c}=\boldsymbol{a} \times \boldsymbol{b}$. In practice, the points forming the head and tails of the spanning vectors are averaged over five adjacent monomers. The correlation time of the autocorrelation function $C_{\boldsymbol{c c}}(t)$ provides a time scale for the internal structural rearrangements of the rings. Correlation times are computed by numerically integrating the normalized $C_{c c}(t)$ from zero to infinity. These times are compared to the diffusion times and the correlation time of the radius of gyration squared in Fig. 5. The longer rings are found to undergo substantial internal rearrangements much faster than they diffuse their own size or relax the overall extension. Not only is the amplitude different, but also the exponent in the power law dependence of $N$ on $\tau_{\text {relax }}$ is significantly lower and approaches a value close to 2.2 . Thus the overall diffusion decouples from the local internal rearrangement, as implied by the decay of $C_{\boldsymbol{c c}}(t)$. This strongly suggests that the slowing down in the mean-square displacement of the overall chain, $g_{3}(t)$, is due to ring-ring coupling, which then also dominates the overall relaxation of $R_{g}^{2}$. For $N \geq 400$ the correlation times of $R_{g}^{2}$ are found to be significantly larger than the internal reorganization times and they closely follow the time scaling of the overall diffusion of the chains. This implies that internal structural 


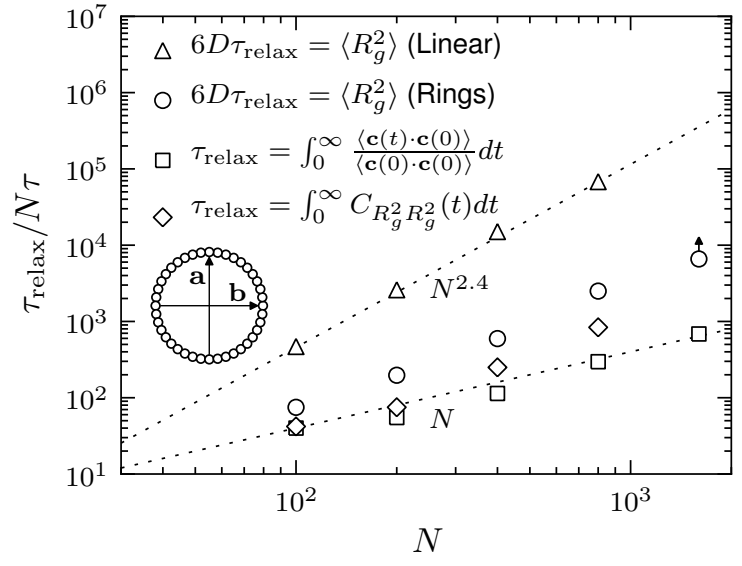

FIG. 5. The diffusive and conformational relaxation times divided by $N$ as a function of chain length. Inset: The $\boldsymbol{a}$ vector is formed between monomers $i$ and $i+N / 2$ while $\boldsymbol{b}$ is formed from $i+N / 4$ and $i+3 N / 4$. With $\boldsymbol{c}=\boldsymbol{a} \times \boldsymbol{b}$, the time scale for single-chain structural relaxation is found by integrating the normalized autocorrelation function of $\boldsymbol{c}$. Despite a very long simulation time it was not possible to accurately determine the correlation time of $C_{R_{g}^{2} R_{g}^{2}}(t)$ for the $N=1600$ ring system. The error bars for the relaxation times of the ring systems with $N<1600$ are smaller than the symbol sizes. The line through the linear chain data is a fit while the $\tau_{\text {relax }} / N \sim N$ line is for reference only.

rearrangement takes place with a fairly small change in the overall extension of the chain.

If the internal reorganization time and the diffusion time are that different, this might also have consequences for the viscosity of the melts. The shear relaxation modulus is

$$
G_{\alpha \beta}(t)=\frac{V}{k_{B} T}\left\langle\bar{\sigma}_{\alpha \beta}(t) \bar{\sigma}_{\alpha \beta}(0)\right\rangle,
$$

where $\bar{\sigma}_{\alpha \beta}(t)$ is the pre-averaged stress 34 . In Eq. 5 . $\alpha$ and $\beta$ are Cartesian indices with $\alpha \neq \beta$, and $V$ is the volume of the simulation box. $G(t)$ is the average of $G_{\alpha \beta}(t)$ with $\alpha \beta=x y, x z, y z$. The shear relaxation modulus is shown in Fig. [6a,b for the linear and ring systems. The linear systems show the characteristic behavior. That is, a Rouse-like decay at early times followed by a plateau region and finally exponential decay. For the rings the behavior is quite different. The ring systems show $G(t) \sim t^{-1 / 2}$ followed by exponential decay. Specifically, the exponent for the stress relaxation tends to increase from -0.5 to -0.45 with increasing $N$. The functional form and exponent for $G(t)$ are nearly the same as those suggested by Kapnistos et al .19 The ring melts are found to relax stress much more rapidly than the linear systems. There is no sign of a plateau even for the largest rings, suggesting that a standard tube model certainly is not applicable. The difference in noise between the two data sets arises from differences in averaging schemes and system sizes (cf. Table 1 of the preceding paper). For the linear chains, the stress was computed
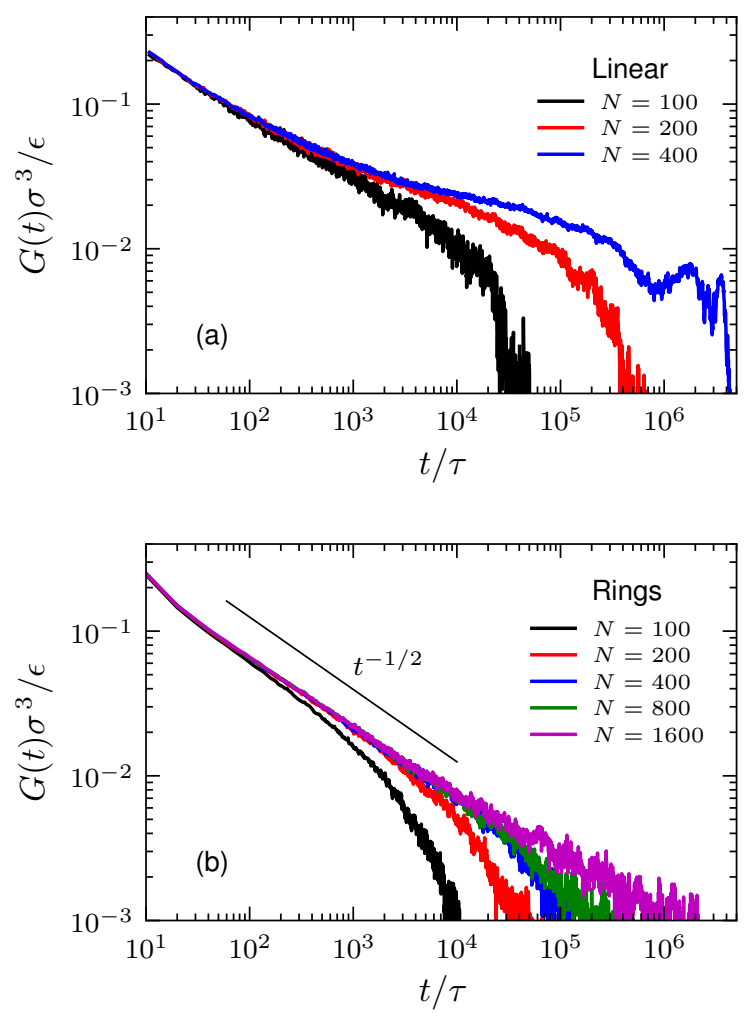

FIG. 6. Stress relaxation for the (a) linear and (b) ring systems. The Rouse scaling is shown for reference in (b). $G(t)$ was computed as described in the main text but additional averaging was performed to make the curves more discernable. The error bars for $G(t)$ increase with increasing $t$.

every other step for 25 steps and averaged for each 100 step interval. For the rings the stress was computed at every step and averaged over each 1000 step interval or $10 \tau$.

Results for the zero-shear viscosity obtained from the Green-Kubo relation ${ }^{35}, \eta_{0}=\int_{0}^{\infty} G(t) d t$, are shown in Fig. 7. The linear systems show a $N^{3.4}$ scaling which is in agreement with previous simulation ${ }^{36}$ and experimental results. ${ }^{37}$ Even though the error bars become quite large for the longer rings and for $N=1600$ we can only give a rough estimate of $\eta_{0}$, the rings show a much weaker dependence on chain length with $\eta_{0} \sim N^{1.4 \pm 0.2}$. For comparison, the Rouse model predicts a linear $\mathrm{N}$ dependence. This is another result which requires a deeper understanding. It appears that stress relaxation, which governs the viscosity, follows a time behavior much closer to the internal reorganization of the rings rather than the overall diffusion and relaxation of $R_{g}^{2}$. The viscosity measurements for the rings and linear systems have been confirmed by nonequilibrium molecular dynamics simulations 38 . These results will appear in an upcoming paper ${ }^{39}$. The zero-shear viscosities are given for the rings and linear systems in Table I.

As an additional check, a simulation was performed 


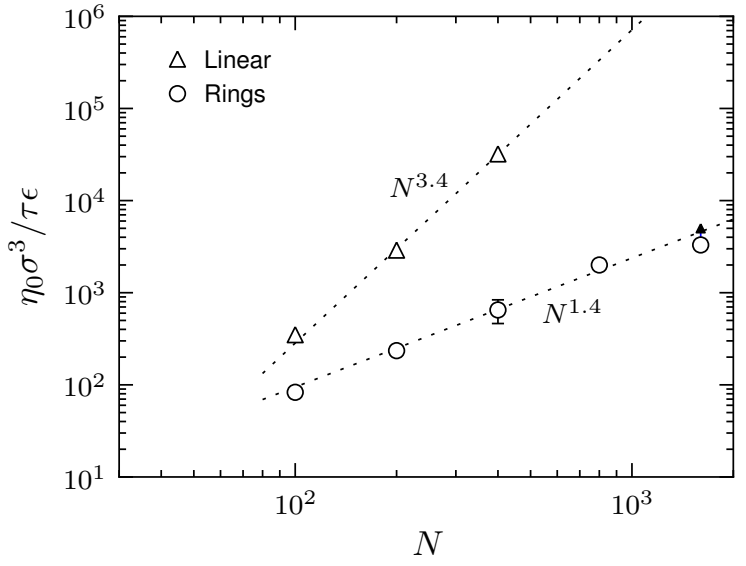

FIG. 7. Zero-shear viscosity of the rings and linear melts versus $N$. The error bars for the $N=400$ ring case were obtained from four independent simulations while the other points were obtained from single simulations. While it is difficult to estimate the error bars for the $N=1600$ case those of the 800 case are expected to be smaller than twice the symbol size. Note the arrow above the data point for the $N=1600$ system.

\begin{tabular}{c|cc|cc}
\hline & \multicolumn{2}{|c|}{ Rings } & \multicolumn{2}{c}{ Linear } \\
\hline$N$ & $D \times 10^{5}$ & $\eta_{0}$ & $D \times 10^{5}$ & $\eta_{0}$ \\
\hline 100 & 38.2 & 83 & 15.4 & 350 \\
200 & 13.0 & 235 & 2.85 & 2900 \\
400 & 3.7 & $650 \pm 180$ & 0.50 & 32100 \\
800 & 0.73 & $\approx 2000$ & 0.11 & - \\
1600 & $<0.23$ & $>3300$ & - & - \\
\hline
\end{tabular}

TABLE I. Transport coefficients for the rings and linear systems. For the same value of $N$ the rings are found to have a higher diffusivity and a lower viscosity. $D$ and $\eta_{0}$ are given in units of $\sigma^{2} / \tau$ and $\tau \sigma^{3} / \epsilon$, respectively. The diffusion coefficient and the viscosity for the $N=1600$ ring case are upper and lower bounds, respectively, since both have not settled down completely within our analysis window. This is partially due to the extensive averaging needed. The error bars for $\eta_{0}$ for the $N=400$ ring case were obtained from four independent simulations.

on the $N=800$ ring system using a dissipative particle dynamics 40141 (DPD) thermostat ${ }^{42}$ instead of the Langevin thermostat. The same friction coefficient was used and $r_{c}$ was taken as $2^{1 / 6} \sigma$. The simulation was run for $8 \times 10^{5} \tau$ and it showed the same behavior for the mean-square displacements indicating that hydrodynamics or momentum conservation are unimportant for these systems on this time scale.

\section{B. Primitive Path Analysis}

Even though ring polymers in a melt cannot entangle in the classical sense, the slowing down of the overall chain diffusion as well as the properties of $g_{1}(t)$ and the slow exchange of neighbors suggest that neighboring rings must be somehow coupled in order to prevent fast free diffusion. Thus an adapted primitive path analysis (PPA) was conducted to elucidate the nature and extent of possible quasi-entanglements in the ring systems. The procedure for linear chains $\frac{13}{13}$ was modified for this purpose. Starting with an equilibrated configuration, a pair of opposite monomers $(i$ and $i+N / 2)$ of each ring polymer are chosen at random to be fixed in space throughout the PPA. With the angular and nonbonded intrachain interactions switched off, the temperature was lowered to $0.05 \epsilon / k_{B}$ and the dynamics were carried out until equilibrium was reached. Without fixed monomers the rings collapse to points. At least three cases were run for each value of $N$.

Fig. 8 shows the last configuration from each PPA simulation for $N=100,400$ and 1600 . For the $N=100$ case, many of the chains have collapsed to double-folded lines with an average contour length, $L_{p p}$, of $2\left\langle R_{e}\right\rangle$. Rings that have not completely collapsed are mutually constrained by one or more neighboring chains. As $N$ increases, the equilibrium fraction of double-folded lines decreases dramatically. For instance, for the $N=1600$ case shown in Fig. 8f all the chains are mutually quasientangled with not a single double-folded line. It can also be seen for increasing chain length that there is a greater tendency for rings to thread or pass through one another. While the chains were initially segregated at the start of the simulation, at equilibrium they are found to form a highly connected melt, even though the volume of each ring is only shared by a small number of other rings.

While somewhat questionable here, the standard argument to deduce an entanglement length for linear chains may be applied to the rings. For the linear chains the entanglement length is $N\left\langle R_{e, \text { linear }}^{2}\right\rangle / L_{p p}^{2}$, which is the number of monomers per Kuhn segment of the primitive path. For the rings this expression is modified by recognizing that a ring should be treated as two linear chains each of length $N / 2$ with common end points. In Fig. 9 we plot $N_{e, \text { rings }}$ against chain length where $N_{e, \text { rings }}=(N / 2)\left\langle R_{e, \text { rings }}^{2}\right\rangle /\left(L_{p p} / 2\right)^{2}$. For the linear chains one finds $N_{e, \text { linear }} \approx 28$, which is independent of $N$ for sufficiently long chains. The entanglement number for the rings is found to vary with chain length with the longest rings giving approximately 77 . This value, however, still seems to slowly grow, which is not totally surprising in view of the early time values of $g_{3}(t) N$ as shown in Fig. 11 and the slow crossover to an asymptotic value of $K_{1}$ (see preceding paper ${ }^{1}$ ). The average contour length for each ring system is given in the inset to Fig. 9. The final configuration from the PPA for the $N=800$ linear system is shown in Fig. 10 for comparison. The entanglement density of the linear system with $N=800$ is higher than that of the $N=1600$ ring system. Our computed value of $N_{e, \text { linear }}$ for this system agrees with the previously reported value of $28 \pm 113$.

These results are to be compared with the fact, found in the previous paper, that the pressure increase due to 


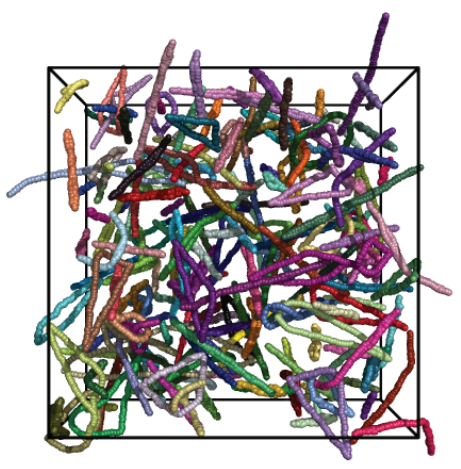

(a) Rings $N=100$

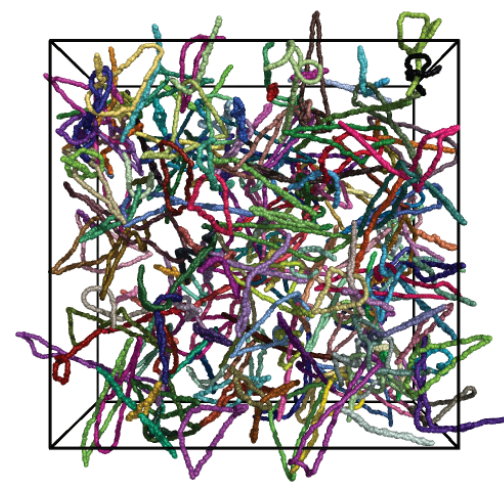

(b) Rings $\mathrm{N}=400$

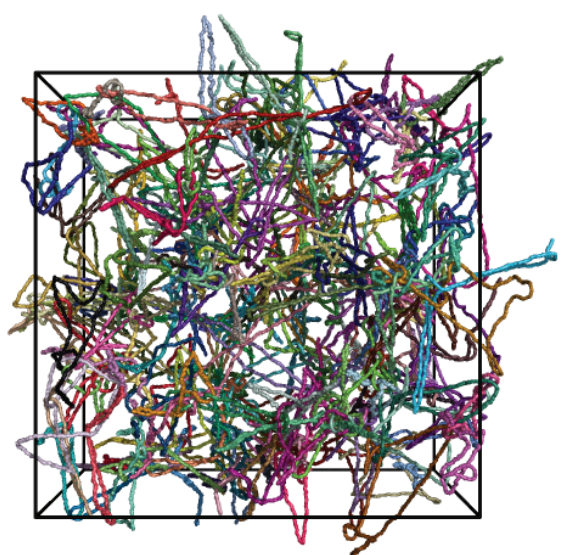

(c) Rings $N=1600$

FIG. 8. Final configurations from a primitive path analysis of the ring systems: (a) $N=100$, (b) 400 and (c) 1600.

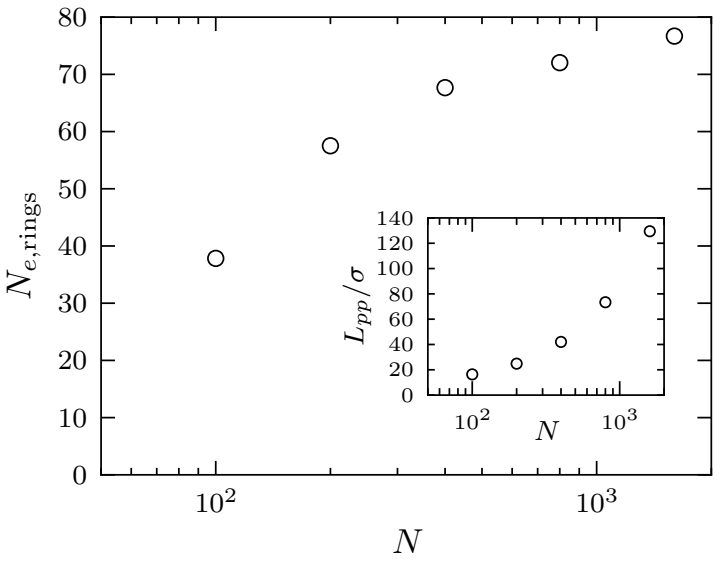

FIG. 9. $N_{e, \text { rings }}$ as a function of chain length. For our model $N_{e, \text { linear }}=28 \pm 1$. The inset gives the average contour length versus $N$.

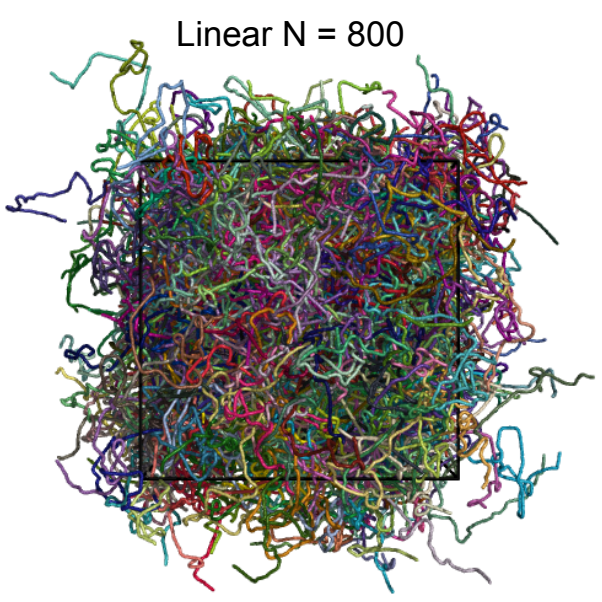

FIG. 10. Same as Fig. 8 except for a linear system with $M=400$ and $N=800$. The linear chains with $N=800$ are far more entangled than the $N=1600$ rings (cf. Fig. 8). the entropy loss associated with the rings squeezing each other in the melt was too small to be observed in our simulations. If the concept of entanglement length $N_{e \text {,rings }}$ makes sense for rings then it should be possible to estimate this pressure increase as $1 / N_{e, \text { rings }}$ times the reference pressure. Given the above estimates of $N_{e \text {,rings }}$ we do not expect to be able to observe such a small difference in pressures.

\section{DISCUSSION}

In the previous paper ${ }^{1}$ we investigated the structural properties of a melt of nonconcatenated rings. It was found that the overall conformations have a number of peculiar properties and are by no means trivial. These peculiar structural properties of the long ring polymer melts have significant consequences for the dynamics. The diffusion of linear chains is explained by the Rouse and reptation theories as demonstrated in the present work. These theories make use of the fact that linear chains have free ends but ring polymers do not have free ends so the application of these theories, and especially reptation theory, is questionable. However, the Rouse model can be solved for a Gaussian ring 22 . The diffusion times from Rouse theory for the linear and ring models are given in Table II. Even for the smallest value of $N$ only fair agreement is found between the simulation results and those predicted by the theory for both architectures. The disagreement grows with increasing $N$ as the systems become more and more entangled, a phenomena which is completely ignored by the theory. The Rouse theory prediction for the viscosity of the rings is in better but also not perfect agreement with the scaling law obtained from simulation.

The peculiar conformational properties of the rings are expected to lead to new dynamic properties. The ring melt simulations give a number of unexpected and not fully understood results. For linear polymers the overall 


\begin{tabular}{c|cc|cc|cc|cc}
\hline & \multicolumn{4}{|c|}{ Rings } & \multicolumn{4}{c}{ Linear } \\
\hline & Rouse Theory & \multicolumn{2}{|c}{ Simulation } & Rouse Theory & \multicolumn{2}{c}{ Simulation } \\
\hline$N$ & $\tau_{D} / 10^{5}$ & $g_{1}\left(\tau_{D}\right)$ & $\tau_{D} / 10^{5}$ & $g_{1}\left(\tau_{D}\right)$ & $\tau_{D} / 10^{5}$ & $g_{1}\left(\tau_{D}\right)$ & $\tau_{D} / 10^{5}$ & $g_{1}\left(\tau_{D}\right)$ \\
\hline 100 & 0.16 & 62.1 & 0.07 & 47 & 0.32 & 94.7 & 0.48 & 78 \\
200 & 0.65 & 125.1 & 0.39 & 91 & 1.29 & 178.3 & 6.77 & 182 \\
400 & 2.59 & 249.8 & 2.38 & 164 & 5.12 & 355.0 & 59.7 & 283 \\
800 & 10.4 & 500.5 & 20.0 & 292 & 20.7 & 714.1 & 559.4 & - \\
1600 & 41.4 & 998.6 & $>105.5$ & - & 82.9 & 1429.2 & - & - \\
\hline
\end{tabular}

TABLE II. Comparison of the diffusion times and mean-square displacement of the monomers between Rouse theory ${ }^{422}$ and the simulation results. The diffusion times are computed using $\tau_{D}=\left\langle R_{g}^{2}\right\rangle / 6 D$. For the Rouse model $D=k T / N \zeta$ for both architectures while $\left\langle R_{g}^{2}\right\rangle$ is $N l_{k} l / 6$ for the linear and $N l_{k} l / 12$ for the rings. To compute $\tau_{D}$ from the simulation data, $D$ is obtained from Table II and $\left\langle R_{g}^{2}\right\rangle$ from Table II of the previous pape ${ }^{1}$. The MD model is mapped to the Rouse model with $l_{k} l=2.71 \sigma^{2}$ and $\zeta=43 \tau^{-1}$. For the linear chain Rouse model $g_{1}\left(\tau_{D}\right)$ was computed using the middle of the chain (i.e., $n=N / 2) . \tau_{D}$ and $g\left(\tau_{D}\right)$ are given in units of $\tau$ and $\sigma^{2}$, respectively.

stress relaxation and the crossover towards free diffusion of the beads is linked to a motion distance of the order of the radius of gyration, even though the center-of-mass motion, $g_{3}(t)$, follows a $t^{1}$ power law at much shorter displacements in comparison to the rings. This is due to the fact that any relaxation is connected to the polymer diffusing out of its original tube. Such a tube concept certainly cannot be applied for rings, at least not directly. Also the strong connection of diffusion distance to stress relaxation does not apply any more here without significant alterations. In short, the dynamics can be summarized by the following observations:

- sub-diffusive behavior of $g_{3}(t) \sim t^{y}$ up to distances 2-3 times $\left\langle R_{g}^{2}\right\rangle$ with $y \approx 0.75$, and a diffusion time $\tau_{D} \sim N^{x}, x \geq 3$ (for $N=1600$ we even find a slowing down $g_{3}(t) \sim t^{y}, y \simeq 0.65$, for larger times),

- the " $t^{1 / 4}$ " regime in $g_{1}(t)$ also extending $2-3$ times $\left\langle R_{g}^{2}\right\rangle$

- diffusion coefficient $D(N) \sim N^{-2.3}$ depends on $N$ in a similar way as in entangled linear polymers, however the prefactor is approximately 7 times larger for the rings with $N=400$ and 800 than the corresponding linear polymers,

- zero-shear viscosity scaling $\eta_{0} \sim N^{1.4 \pm 0.2}$, which is much more suggestive of Rouse-like behavior $(\sim$ $N)$ than reptation $\left(\sim N^{3.4}\right)$, is in accord with an internal reorganization time $\mathcal{O}\left(N^{2.2 \pm 0.1}\right)$.

The first three points seem to contradict the fourth which suggests that the overall motion of the chains and the stress relaxation, at least to within our accuracy, are somewhat decoupled. In the following we propose a mechanism which can account for this. Also, the further slowing down of $g_{3}(t)$ for $N=1600$ is required in order to prevent an early, unphysical crossing of $g_{1}(t)$ and $g_{3}(t)$ (see below).

At short times $\left(t \lesssim \tau_{e}\right)$ we expect the motion of the beads, $g_{1}(t)$, to follow the standard Rouse $t^{1 / 2}$ behavior, and as shown in Fig. 1 this is indeed found. This is in contrast to $g_{3}(t)$ which does not follow the standard Rouse behavior as discussed above. Once the entanglement time is reached, a slowing down at a motion amplitude of the order of the tube diameter $d_{T}^{2} \approx b^{2} N_{e \text {,linear }}$ is expected. This is observed for both the linear and ring polymers. Then one expects for linear polymers the Rouse motion along the tube, leading to the well-known $t^{1 / 4}$ regime and then, after the Rouse time, the diffusion out of the tube, resulting in the second $t^{1 / 2}$ regime, until after the disentanglement time $\tau_{d} \sim N^{3.4}$ where the free diffusion dominates. For rings we expect a similar initial slowing down due to the motion along the short branches of the lattice animal-like structure (shorter rings) or of the crumpled globule (large rings). It appears, however, that this slowed down motion and the diffusion along the chain contour itself, as suggested by the decay of the autocorrelation function of the cross product of the spanning vectors, cf. Fig. 5, create a unique power law very close to $t^{1 / 4}$ over a very long time scale and distances exceeding the gyration radius of the rings. Then there is a crossover towards free diffusion without an additional clearly-defined power law in between, which is in contrast to the behavior of linear chains.

Although we do not have a consistent theoretical explanation for the observed dynamics, we can formulate an argument relating some of our observations to the others. Specifically, the slowed down sub-diffusive motion of the beads requires some reduction in the effective exponent of the center-of-mass motion as well, otherwise $g_{1}(t)$ and $g_{3}(t)$ would meet or even cross (meaning that the coil as a whole would move much further than a monomer, which is obviously impossible) at a time even well below the Rouse time. Let us assume $g_{3}(t) \sim t^{\alpha_{3}}$ for the "chain diffusion" at intermediate times and $g_{1}(t) \sim t^{\alpha_{1}}$ for one monomer diffusion. We might write for a time dependent "diffusion constant" $D\left(g_{1}(t)\right) t^{\alpha_{3}}=g_{1}(t)$. Assuming that $D\left(g_{1}(t)\right) \sim g_{1}(t)^{-d_{f} / 2}$, namely proportional to the number of beads carried along at a distance $g$ within an object of fractal dimension $d_{f}=3$, we arrive at the relation $\alpha_{3}=(5 / 2) \alpha_{1} \underline{43}$. This relation appears to be consistent with our simulation data. Indeed, careful 
inspection of the data for $g_{1}(t)$ indicates that the smallest slope is close to 0.28 , systematically decaying with increasing chain length to slightly larger than $1 / 4$. The smallest slope in $g_{3}(t)$ for $N=1600$ is close to 0.7 , which is in good agreement with the above relation between $\alpha_{1}$ and $\alpha_{3}$.

Also the amplitude of $g_{3}(t)$, where the crossover towards free diffusion occurs needs some more attention. For instance, as shown in Fig. 11, for the $N=400 \mathrm{ring}$ case the transition to $g_{3}(t) \sim t^{1}$ at long times occurs at roughly $7.1 \times 10^{5} \tau$ where $g_{3}(t)=170 \sigma^{2}$ or $3.2\left\langle R_{g}^{2}\right\rangle$. For the linear case at the transition one finds $g_{3}(t)=170 \sigma^{2}$ or $0.9\left\langle R_{g}^{2}\right\rangle$. For the ring systems with $N=400$ and 800 (and extrapolated for $N=1600$ ) one then finds $D \sim N^{-2.3}$. All previous simulation papers have reported a weaker dependence with the strongest by Hur et al. 23 being $D \sim N^{-1.9}$. This is most probably due to the use of effectively shorter chains in the previous studies.

Another surprising result regarding the diffusion of the rings is that the stress relaxation follows an approximate $t^{-1 / 2}$ scaling law, in fair agreement with the $t^{-2 / 5}$ prediction of Kapnistos et al! ${ }^{[19}$ In fact, the $N=800 \mathrm{ring}$ system shows this behavior for at least four decades in time. While it is tempting to appeal to the Rouse model since it predicts $G(t) \sim t^{-1 / 2}$, this certainly cannot apply. This fast decay, compared to what we observe from the mean-square displacements of the beads, is in accord with the observation that $C_{c c}(t)$ decays with a relaxation time $\tau_{\text {relax }} \sim N^{2.2 \pm 0.1}$. That means stress can relax by internal reorganization without the need to diffuse a significant distance away from its original neighbor chains or to fully relax the overall shape of the chain. This however is only possible because the rings eventually are compact objects. As a consequence, the characteristic stress relaxation time $\tau_{\text {relax }} \sim N^{2.2}$ implies that the very small viscosity $\eta_{0}$ and the diffusion time, $\tau_{D} \sim N^{x}, x \geq 3$, are not directly related to each other. This line of arguments does not take into account the existence of, as we called them, quasi-entanglements, which relax only very slowly and might give rise to a plateau-like regime in the stress relaxation function. For a possible plateau, however, we expect a very small value of the order $\mathcal{O}\left(k_{B} T / N\right)$, simply because the number of neighbors that a given ring can entangle with becomes independent of $N$ and settles at a value below 20, a typical number for a liquid. Thus, if existing, such a plateau will be very difficult to observe and beyond the accuracy of our data.

An attempt to describe the amoeba-like motion of the individual ring among other nonconcatenated rings was presented in a recent article by Milner and Newhall27. These authors argue that since the ring in the lattice of obstacles passes each lattice gate twice in the opposite directions and, therefore, represents a kind of tree, then each bond separates the whole system in two halves, with the sizes $k$ and $N-k$, respectively. The smaller of the two numbers Milner and Newhall call "centrality" and provide convincing simulation data to the effect that effective motion along the "reaction coordinate" of central- ity is sub-diffusive with $\delta k \sim t^{3 / 4}$. Clearly, the diffusion of centrality in this picture is also closely related to the stress relaxation, which means that this picture is ideologically very close to that proposed by Kapnistos et a19. Given the identical values of the exponents in the diffusion of centrality case and in our $g_{3}(t)$ behavior for intermediate times and ring sizes, it is tempting to bridge the two statements. However, our conformations somehow suggest a rather extended regime of beads to qualify for the centrality argument, making the precise meaning of this concept somewhat unclear here. Future work will be required to understand whether they are really related or there is just a coincidence of numerical values for the two exponents.

\section{CONCLUSIONS}

The structural and dynamic properties of ring polymer melts have been investigated by molecular dynamics simulation using a semiflexible bead-spring model in this and the previous paper. For the longest chain lengths, the radius of gyration was found to approach a scaling regime of $N^{1 / 3}$ in agreement with previous simulation results and theoretical studies. While this suggests collapsed conformations, the rings are, in fact, open or expanded and highly interpenetrated by their few $\mathcal{O}\left(N^{0}\right)$ neighbors. This is supported by a primitive path analysis which was also used to show that the estimated quasi-entanglement number of the rings is larger than that of the analogous linear chains. The rings being not as entangled as the linear chains and more compact in size may explain why they relax stress faster and have a viscosity which scales much more weakly than the $N^{3.4}$ dependence of linear chains. Despite following different scaling laws with $N$ for the radius of gyration, stress relaxation and viscosity, and undergoing different modes of motion while diffusing, for large $N$ the two architectures approximately obey the same $D \sim N^{-2.3 \pm 0.1}$ power law. Because of the compact nature of the ring conformations, resulting in a fairly deep correlation hole, obtaining diffusion constants from such simulations might be subject to rather strong finite size effects in the number of chains considered. Such polymeric systems have some resemblance to a soft sphere liquid which is known to be subject to such effects. However, we are confident that our systems with $M=200$ ring polymers are sufficiently large and we emphasize that studies using significantly fewer chains should be avoided.

Though we have presented a rather comprehensive analysis many questions remain open. Conformational properties need further attention, especially for questions with respect to biological issues. This concerns, for example, contact maps and the life time of the clusters seen in such maps 1 . Also, the precise determination of $\beta$ and $\gamma$ and the related scaling prediction $\beta+\gamma=2$ need further attention. The decoupling of translational motion and stress relaxation for rings, the latter leading 
to the low viscosity, certainly is not fully understood and needs further investigation. The sub-diffusive regime in the MSD of the rings up to displacements that are significantly larger than $\left\langle R_{g}^{2}\right\rangle$ poses special challenges for a proper theoretical description. To better compare our findings to experiments, a systematic study of the influence of linear "contaminants" is needed since experiments never can exclude them completely. Such a study is in preparation ${ }^{39}$ and will also include an extensive analysis of the dynamic scattering functions of both the rings and the linear polymers. Recent advances in synthesis, purification and characterization should allow a better comparison in the near future. While the main thrust of the two papers was on the properties of nonconcatenated ring polymer melts, for comparison we also have presented extensive simulations of highly entangled melts of linear polymers. While in general the reptation picture provides a good qualitative and semi-quantitative theoretical framework there are still open questions with respect to the slow crossover to the anticipated asymptotic regime or possible deviations thereof.

\section{ACKNOWLEDGMENTS}

The authors are grateful to T. Vilgis, D. Fritz and V. Harmandaris for their comments on an early version of the manuscript. The ESPResSo development team is acknowledged for optimizing the simulation software on the IBM Blue Gene/P at the Rechenzentrum Garching in Münich, Germany. We thank Donghui Zhang for discussions and references relating to experimental studies on cyclic polymers. This project was in part funded by the Alexander von Humboldt Foundation through a research grant awarded to AYG. AYG also acknowledges the hospitality of the Aspen Center for Physics where part of this work was done. WBL acknowledges financial support from the Alexander von Humboldt Foundation and the Basic Science Research Program through the National Research Foundation of Korea (NRF) funded by the Ministry of Education, Science and Technology (2010-0007886). Additional funding was provided by the Multiscale Materials Modeling (MMM) initiative of the Max Planck Society. We thank the New Mexico Computing Application Center NMCAC for a generous allocation of computer time. This work is supported by the Laboratory Directed Research and Development program at Sandia National Laboratories. Sandia is a multiprogram laboratory operated by Sandia Corporation, a Lockheed Martin Company, for the United States Department of Energy under Contract No. DE-AC04-94AL85000.

${ }^{1}$ J. D. Halverson, W. Lee, G. S. Grest, A. Y. Grosberg, and K. Kremer, J. Chem. Phys., DOI: 10.1063/1.3587137 (2011).

${ }^{2}$ P. de Gennes, J. Chem. Phys. 55, 572 (1971).

${ }^{3}$ P. de Gennes, Scaling Concepts in Polymer Physics (Cornell University Press, 1979).

${ }^{4}$ M. Doi and S. F. Edwards, The Theory of Polymer Dynamics (Oxford University Press, 1986).
${ }^{5}$ A. V. Tobolsky, Properties and Structure of Polymers (Wiley, New York, 1960).

${ }^{6}$ S. Onogi, T. Masuda, and K. Kitagawa, Macromolecules 3, 109 (1970).

${ }^{7}$ G. C. Berry and T. G. Fox, Adv. Polym. Sci. 5, 261 (1968).

${ }^{8}$ A. Casale, R. S. Porter, and J. F. Johnson, J. Macromol. Sci. Rev. Macromol. Chem. Phys. C5, 387 (1971).

${ }^{9}$ H. Odani, N. Nemoto, and M. Kurata, Bull. Inst. Chem. Res. Kyoto Univ. 50, 117 (1972).

${ }^{10}$ K. Kremer and G. S. Grest, J. Chem. Phys. 92, 5057 (1990).

${ }^{11} \mathrm{~K}$. Binder, Monte Carlo and Molecular Dynamics Simulations in Polymer Science (Oxford University Press, 1995).

${ }^{12}$ T. C. B. McLeish, Advances in Physics 51, 1379 (2002).

${ }^{13}$ R. Everaers, S. K. Sukumaran, G. S. Grest, C. Svaneborg, A. Sivasubramanian, and K. Kremer, Science 303, 823 (2004).

${ }^{14} \mathrm{~J}$. Roovers and P. M. Toporowski, Macromolecules 16, 843 (1983).

${ }^{15}$ G. Hild, C. Strazielle, and P. Rempp, Eur. Polym. J. 19, 1983 (1983).

${ }^{16}$ G. B. McKenna, G. Hadziioannou, P. Lutz, G. Hild, C. Strazielle, C. Straupe, and P. Rempp, Macromolecules 20, 498 (1987).

${ }^{17}$ J. Roovers and P. M. Toporowski, J. Polym. Sci. B 26, 1251 (1988).

${ }^{18}$ S. F. Tead, E. J. Kramer, G. Hadziioannou, M. Antonietti, H. Sillescu, P. Lutz, and C. Strazielle, Macromolecules 25, 3942 (1992).

${ }^{19}$ M. Kapnistos, M. Lang, D. Vlassopoulos, W. Pyckhou-Hintzen, D. Richter, D. Cho, T. Chang, and M. Rubinstein, Nature Materials 7, 997 (2008).

${ }^{20}$ S. Nam, J. Leisen, V. Breedveld, and H. W. Beckham, Macromolecules 42, 3121 (2009).

${ }^{21}$ K. Hur, R. G. Winkler, and D. Y. Yoon, Macromolecules 39, 3975 (2006).

${ }^{22}$ G. Tsolou, N. Stratikis, C. Baig, P. S. Stephanou, and V. G. Mavrantzas, Macromolecules 43, 10692 (2010).

${ }^{23}$ K. Hur, C. Jeong, R. G. Winkler, N. Lacevic, R. H. Gee, and D. Y. Yoon, Macromolecules 44, 2311-2315 (2011).

${ }^{24}$ S. K. Sukumaran, G. S. Grest, K. Kremer, and R. Everaers, J Polym Sci Part B: Polym Phys 43, 917 (2005).

${ }^{25}$ I. Carmesin and K. Kremer, J. de Physique 51, 915 (1990).

${ }^{26}$ J. P. Wittmer, H. Meyer, A. Johner, T. Kreer, and J. Baschnagel, Phys. Rev. Lett. 105, 037802 (2010).

${ }^{27}$ S. T. Milner and J. D. Newhall, Phys. Rev. Lett. 105, 208302 (2010)

${ }^{28}$ See ancillary files for the mean-square displacement data of the rings and linear systems as well as the various relaxation times of the rings.

${ }^{29}$ For very short times, below the ones shown here, $g_{3}(t) N$ is independent of $N$ and $\sim t^{1}$. Thus the observed behavior indicates that the initial free diffusion extends to slightly larger distances for the longer rings, reminiscent of typical cage effects in liquids.

${ }^{30}$ M. Müller, J. P. Wittmer, and M. E. Cates, Phys. Rev. E 53, 5063 (1996).

${ }^{31}$ S. Brown and G. Szamel, J. Chem. Phys. 109, 6184 (1998).

${ }^{32}$ M. Müller, J. P. Wittmer, and M. E. Cates, Phys. Rev. E 61, 4078 (2000).

${ }^{33}$ T. P. Lodge, Phys. Rev. Lett. 83, 3218 (1999).

${ }^{34}$ W. Lee and K. Kremer, Macromolecules 42, 6270 (2009).

${ }^{35}$ M. P. Allen and D. J. Tildesley, Computer Simulation of Liquids (Oxford Science Publications, 1987).

${ }^{36}$ M. Kröger and S. Hess, Phys. Rev. Lett. 85, 1128 (2000).

${ }^{37}$ J. D. Ferry, Viscoelastic Properties of Polymers (Wiley, 1980).

${ }^{38}$ M. E. Tuckerman, C. J. Mundy, S. Balasubramanian, and M. L. Klein, J. Chem. Phys. 106, 5615 (1997).

${ }^{39}$ J. D. Halverson, G. S. Grest, A. Y. Grosberg, and K. Kremer, in preparation.

${ }^{40}$ P. J. Hoogerbrugge and J. Koelman, Europhysics Letters 19, 155 (1992).

${ }^{41}$ P. Espanol and P. Warren, Europhysics Letters 30, 191 (1995).

${ }^{42}$ T. Soddemann, B. Dünweg, and K. Kremer, Phys. Rev. E 68, 
046702 (2003).

${ }^{43}$ For a Rouse chain this argument shows that $g_{3}(t) \sim t$ requires $g_{1}(t) \sim t^{1 / 2}$ for $g_{3}(t)<\left\langle R_{e}^{2}\right\rangle$. 